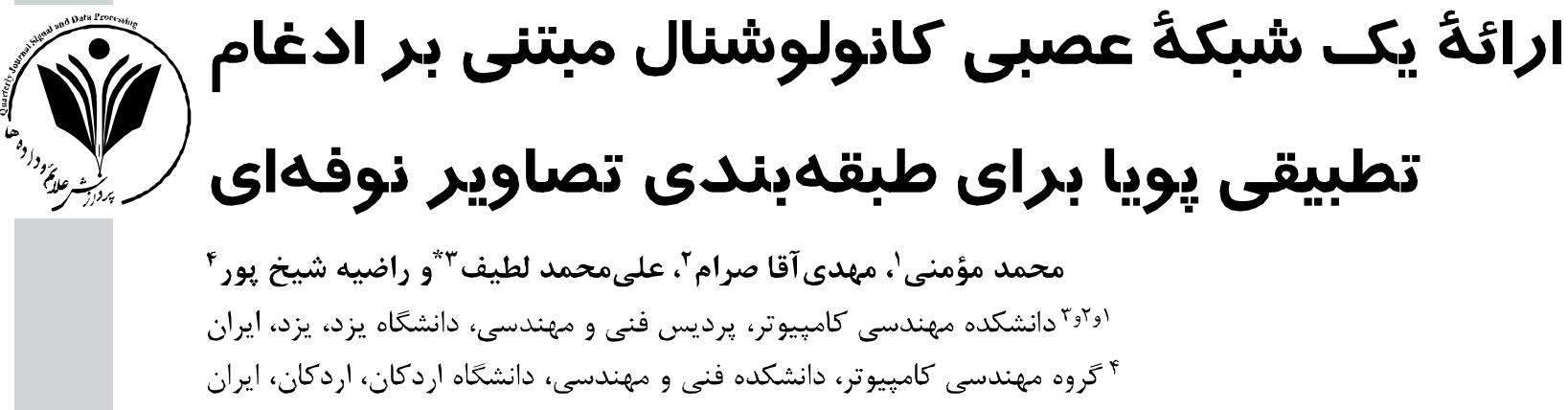

جكبده

طبقابندى تصاوير مبتنى بر شبكه عصبى كانولوشن (CNN) بهصورت كسترده در حوزه بينايى ماشين مورد مطالعه قرار كرفته است. تصاوير

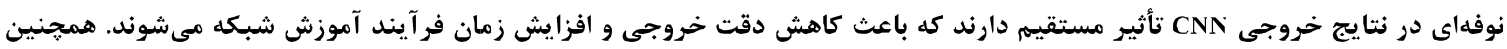

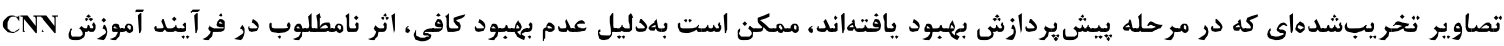

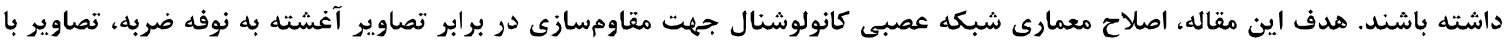

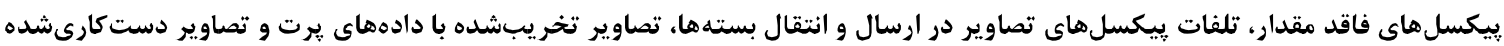

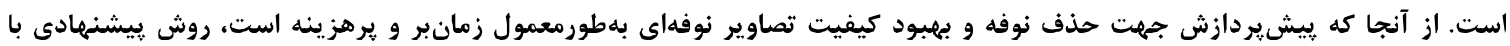

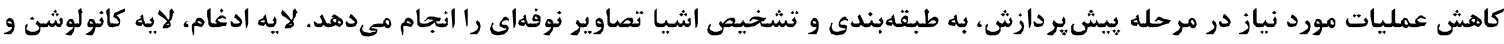

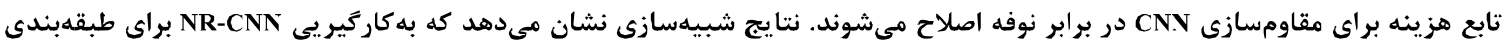

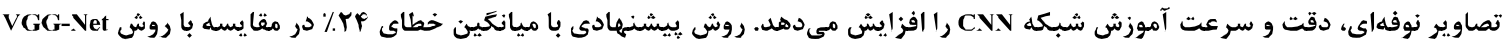

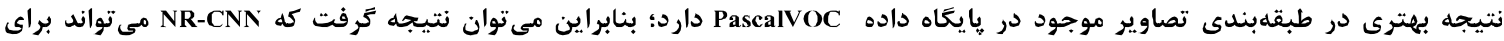
طبقلبندى و تشخيص شىء در تصاوير نوفهاى سودمند باشد.

وازَٔان كليدى: شبكه عصبى كانولوشنال، نوفه، طبقهبندى تصوير، ادغام تطبيقى، ادغام وزندار

\title{
A Convolutional Neural Network based on Adaptive Pooling for Classification of Noisy Images
}

\author{
Mohammad Momeny ${ }^{1}$, Mehdi Agha Sarram², AliMohammad Latif ${ }^{3 *}$ \\ \& Razieh Sheikhpour ${ }^{4}$ \\ 1,2,3 Department of Computer Engineering, Faculty of Engineering, \\ Yazd University, Yazd, Iran \\ ${ }^{4}$ Department of Computer Engineering, Faculty of Engineering, \\ Ardakan University, Ardakan, Iran
}

\begin{abstract}
Convolutional neural network is one of the effective methods for classifying images that performs learning using convolutional, pooling and fully-connected layers. All kinds of noise disrupt the operation of this network. Noise images reduce classification accuracy and increase convolutional neural network training time. Noise is an unwanted signal that destroys the original signal. Noise changes the output values of a system, just as the value recorded in the output differs from its actual value. In the process of image encoding and transmission, when the image is passed through noisy transmission channel, the impulse noise with positive and negative pulses causes the image to be destroyed. A positive pulse in the form of white and

* Corresponding author

* تويسنده عهدهار مكاتبات

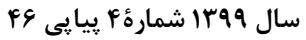

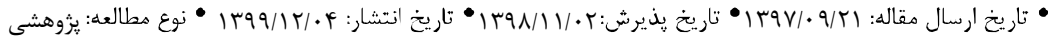


a negative pulse in the form of black affect the image. The purpose of this paper is to introduce dynamic pooling which make the convolutional neural network stronger against the noisy image. The proposed method classifies noise images by weighting the values in the dynamic pooling region. In this research, a new method for modifying the pooling operator is presented in order to increase the accuracy of convolutional neural network in noise image classification. To remove noise in the dynamic pooling layer, it is sufficient to prevent the noise pixel processing by the dynamic pooling operator. Preventing noise pixel processing in the dynamic pooling layer prevents selecting the amount of noise to be applied to subsequent CNN layers. This increases the accuracy of the classification. There is a possibility of destroying the pixels of the entire window in the image. Due to the fact that the dynamic pooling operator is repeated several times in the layers of the convolutional neural network, the proposed method for merging noise pixels can be used many times. In the proposed dynamic pooling layer, pixels with a probability of $p$ being destroyed by noise are not included in the dynamic pooling operation with the same probability. In other words, the participation of a pixel in the dynamic pooling layer depends on the health of that pixel value. If a pixel is likely to be noisy, it will not be processed in the proposed dynamic pooling layer with the same probability. To compare the proposed method, the trained VGG-Net model with medium and slow architecture has been used. Five convolutional layers and three fully connected layers are the components of the proposed model. The proposed method with $26 \%$ error for images corrupted with impulse noise with a density of $5 \%$ has a better performance than the compared methods. Increased efficiency and speed of convolutional neural network based on dynamic pooling layer modification for noise image classification is seen in the simulation results.

Keywords: Convolutional neural network, Noise, Image classification, weighted pooling

$$
\begin{aligned}
& \text { با پالس مثبت و منفى موجب تخريب تصوير مىشود. يالس }
\end{aligned}
$$

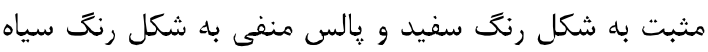

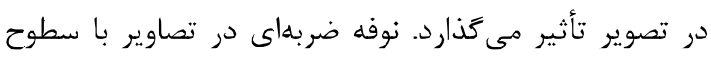

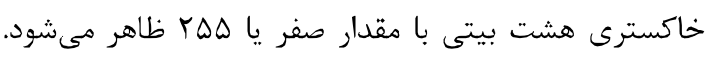

$$
\begin{aligned}
& \text { نوفه ضربهاى (دو قطبى) بهصورت زير مشخص مىشود }
\end{aligned}
$$

$p(z)=\left\{\begin{array}{c}P_{a} \quad z=a \\ P_{b} \quad z=b \\ 0 \text { Other Wise }\end{array}\right.$

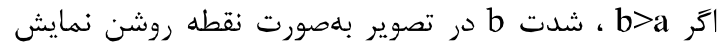

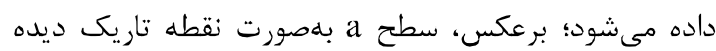

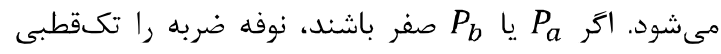

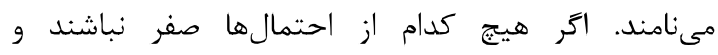
بهخصوص اخر آنها بهطورتقريبى مساوى باشند، مقادير نوفه

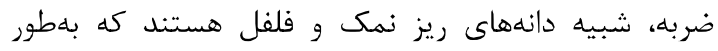

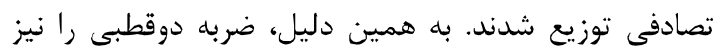
نوفه نمك و فلفل گويند علاوهبراين از وازههاى نوفه لكه و

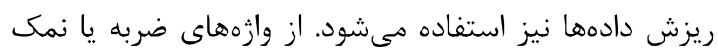

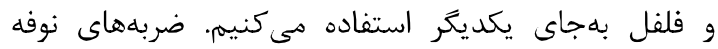

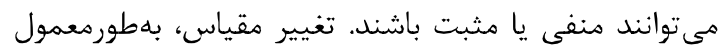

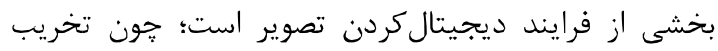

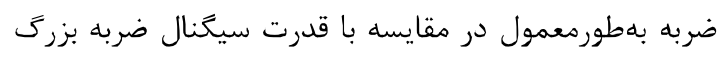
است، نوفه ضربه باطور كلى بهصورت مقادير حدى (سفيد يا

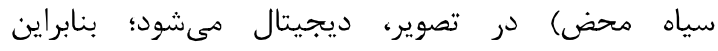
بهطورمعمول فرض بر اين است كه a و b مقادير اشباعشده هستند؛ زيرا برابر با مقادير مجاز بيشينه و كمينه در در تصوير

$$
\text { مان }
$$

در فرايند رمزكذارى و انتقال تصوير، زمانى كه تصوير

از خطوط انتقالى داراى نوفه عبور داده مىشود، نوفه ضربهائ

${ }^{1}$ Convolutional Neural Network

${ }^{2}$ Missing samples

${ }^{3}$ Packet loss in image transmission

${ }^{4}$ Damaged image

${ }^{5}$ Tampered images 
عصبى كانولوشنال را افزايش دهد. نتايج شبيهسازى، مشخص كننده كاهش نرخ خطا و افزايث سرعت در در طبقابندى است. اهميت تعيين ديزان دقيق تزريق نوفه به تصوير در افزايش سرعت طبقلبندى از معايب اين روش

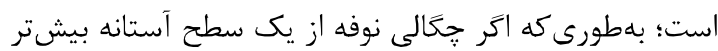

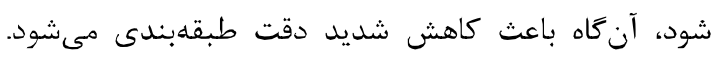
يِيشيردازش يكى از روشهاى رفع اختلالات و نوفه است.

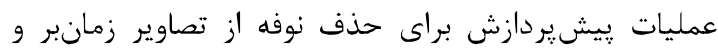

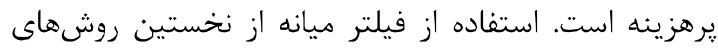
حذف نوفه ضربه است. از معايب روشهاى مبنته استى بر فيلتر ميانه اين است كه اكر تصوير داراى نوفه با جَّالى بالا باشد،

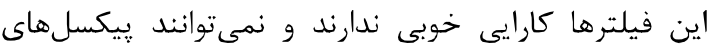
بدون نوفه را از ييكسلهاى نويزى تشخيص دهند و اين منجر به حذف اطلاعات مهرم تصوير مىشود [20]. اساكيراجانه و همكارانش براى بازيابى تصاوير با نوفه ضربه،

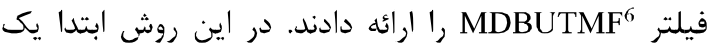

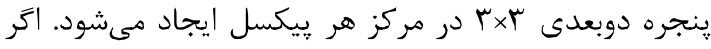

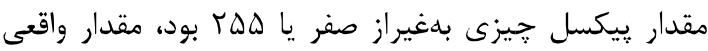
ييكسل شناختهشده و زيردازش نمىشود. اكر كليه مقادير

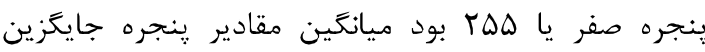

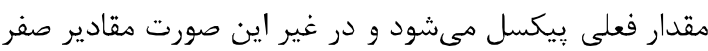

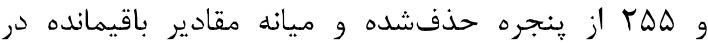

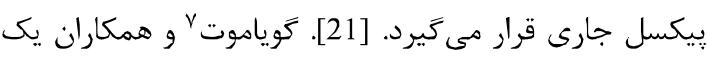
روش مبتنى بر معادلات ديفرانسيل با مشتقات جزئى براى بهبود تصاوير تخريبشده ارائه دادند [22].

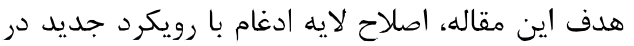
معمارى شبكه CNN براى افزايش دقت طبقدبندى تصاوير

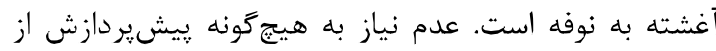
مزاياى روش بيشنههادى است.

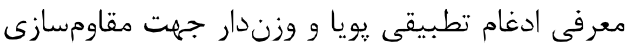
CNN آزمايشها در بخش سوم ارائه مىشود. بخش حهارم شامل

$$
\text { نتيجه كيرى است. }
$$

\section{r- با روش بيشنهادى}

در اين بخش معمارى روش ييشنهادى با توجه به روندنماى

$$
\text { شكل (1) تشريح مىشود. }
$$

${ }^{5}$ Esakkirajan

${ }^{6}$ Modified Decision Based Unsymmetric Trimmed Median ${ }^{7}$ Guillemot
ديجيتال شده هستند؛ در نتيجه، ضربههاى منفى بلهصورت

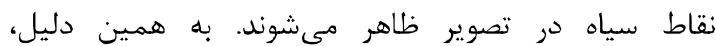
ضربههاى مثبت بهصورت نوفه سفيد ظاهر مىشود. براى

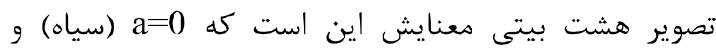
b=255 (سفيد) است نوفه ضربهاى در وضعيتهايى زييدا

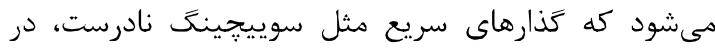

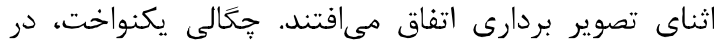
وضعيتهاى عملى كمتر اتفاق هىافتد؛ اما شدت يكنواخت بهعنوان مبنايى براى مولدهاى اعداد تصادفى محسوب

مىشود كه در شبيهسازى به كار مى آيند [14]-[17].

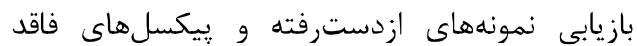
مقدار در بسيارى از زمينههاى يردازش سيخنال مورد بررسى بـى بهى

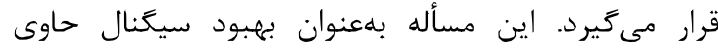
اطلاعات ناقص شناخته مىشود [18] و [19]. استفاده از شبكهاى حسكر بىسيم نيز يكى از دلايل اتلاف مقادير

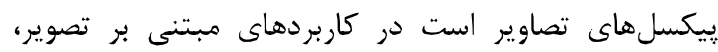
حجم دادههايى كه بايد منتقل شوند، بيشتر از ساير برنامهاي هستند؛ بنابراين، خطاها و تلفات بسته در دهن هنغام انتقال

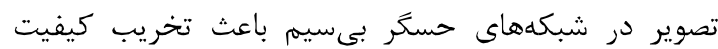
تصوير مى شوند [20] و [21].

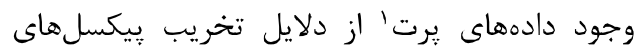

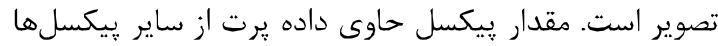
فاصله زيادى دارد. در فرايند اكتساب تصوير، ارسال تصوير و

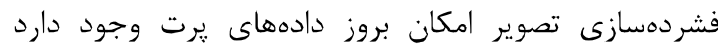
[22] و [23]. يكى ديگر از الكوهاى تخريب تصاوير، دست كارى عمدى است. بازيابى بخشهاى دست كارى لشده

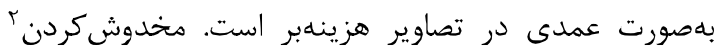
عمدى تصاوير از علل كاهش كيفيت تصوير است. [24] و

شناسايى نوع نوفه توسط شبكه عصبى كانولوشنال مكانيذير است، ولى مقاومسازى شبكه در برابر نوفه هنوز

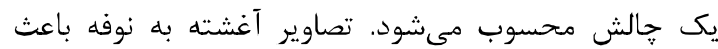
كاهش دقت طبقلبندى در شبكه عصبى كانولوشنال مىشود و بروز هركونه اختلال در تصاوير ورودى CNN در نتايج

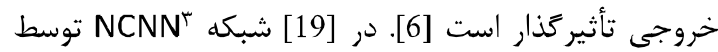
اودخاسى † و همكاران sعرفى و نشان داده شد كه تزريق نوفه به تصوير در شرايط خاص مىتواند سرعت آموزش شبكه

${ }^{1}$ Outlier

${ }^{2}$ Scratch

${ }^{3}$ Noisy Convolutional Neural Network

${ }^{4}$ Audhkhasi 
فرايند آموزش مبتنى بر نقشه نوفه، روش بيشنهادى نحوه

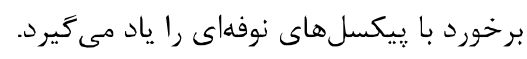

$$
\text { r-Y - Fام تطبيقى در برابر نوفه }
$$

الكوريتم ييشنهادى براى تنظيم نحوه كام تطبيقى در برابر

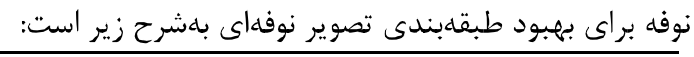

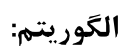

1- ساخت يك ماتريس بيتى بلعنوان نقشه كام

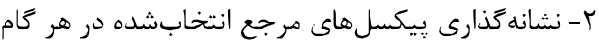

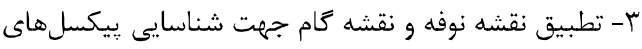

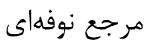

f- تغيير موقعيت فيلتر از نواحى نوفهاى به نزديكترين موقعيت

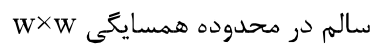

ا-Y- - لايه شناسايى نوفه

وظيفه لايه شناسايى نوفه، تهيه نقشه نوفه است تالت

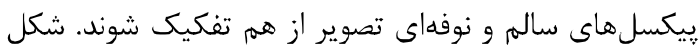

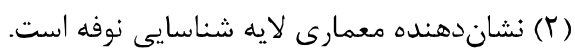
نقشه نوفه در اين مقاله، بيانخر دستهبندى مقادير

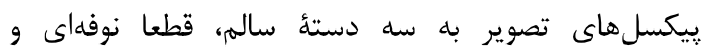

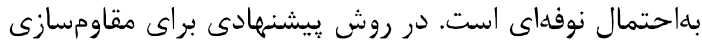
CNN

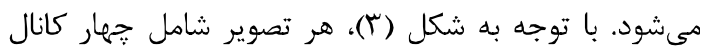

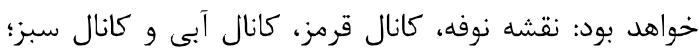

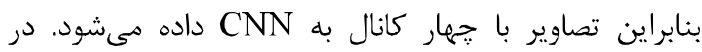
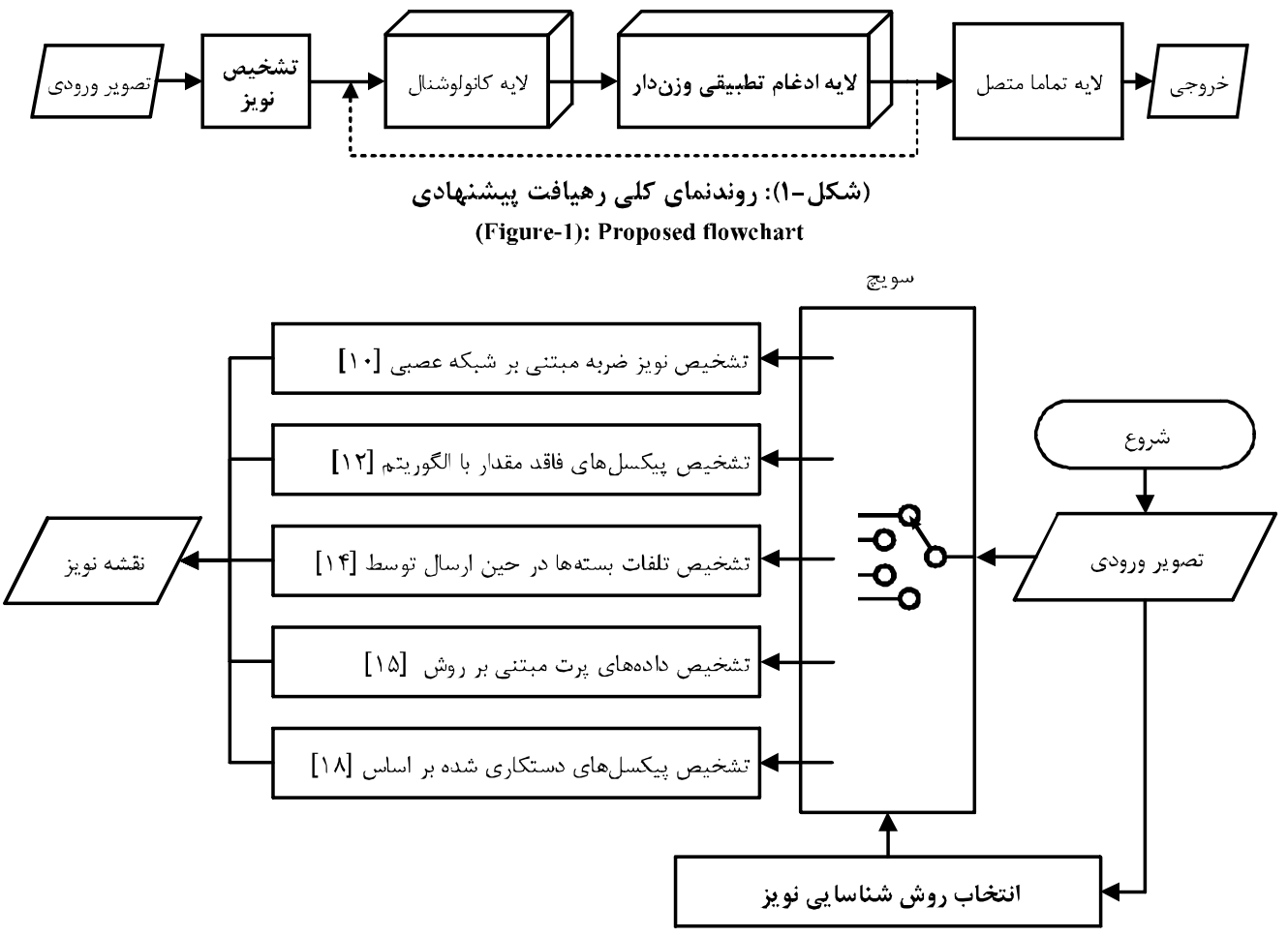

(شكل -Y): لايه شناسايى نوفه

(Figure-2): Noise detection layer
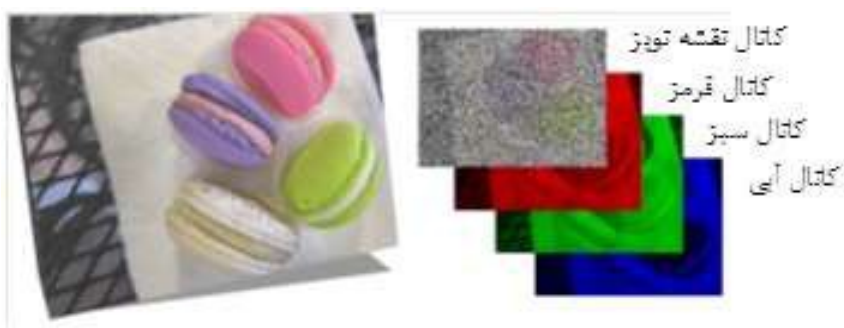

(شكل-r): جهار كانال براى هر تصوير

(Figure-3): Four channel for each image 
نزديكترين موقعيت سالم براى تغيير موقعيت فيلتر جستجو

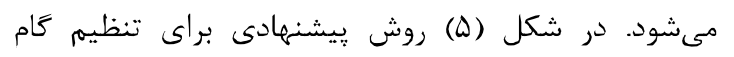

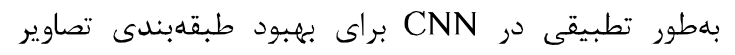
نوفهاى نشان داده شده است.

اصلاح نقشه كام بلطور تطبيقى باعث عدم شركت

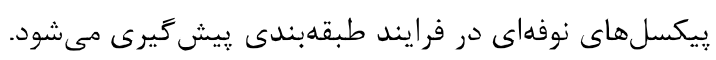

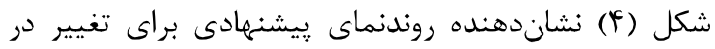

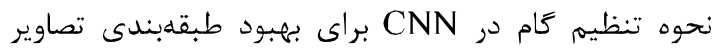
آغشته به نوفه است. W×W محدودماى است كه براى يافتن

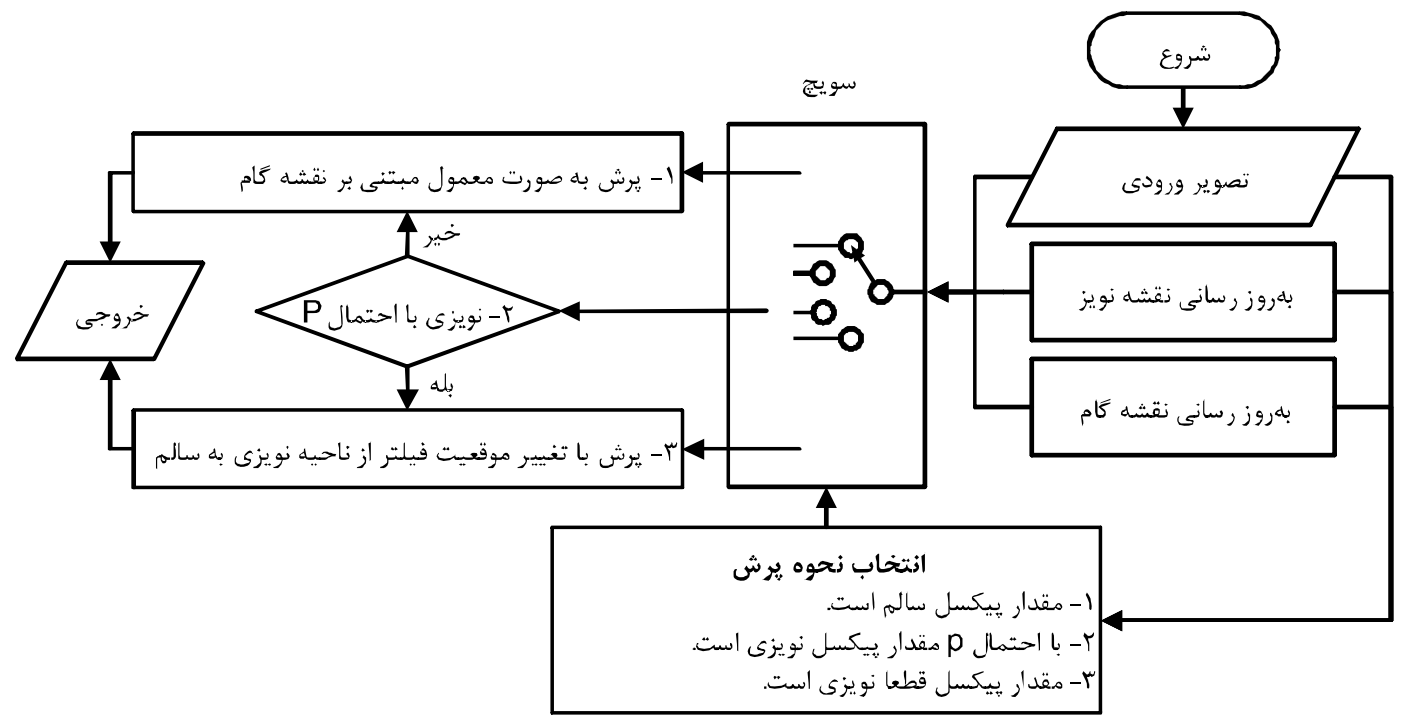

(شكل -F): روندذماى كام تطبيقى در برابر نوفه. ابتدا نقشه نوفه و نقشه كام باهم مطابقت داده شده و سبِ برش انجام ميىشود. (Figure-4): The workflow of the proposed adaptive stride to noise

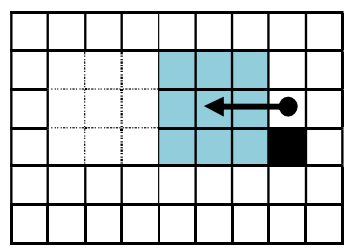

ج: تغيير موقعيت فيلتر از نواحى نوفهاى به نزديكترين موقعيت سالم

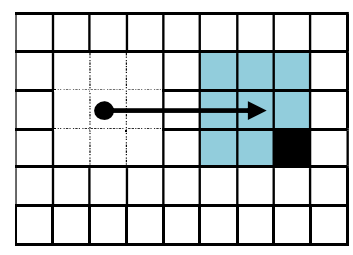

ب: تطبيق نقشه نوفه و نقشه كام جهت

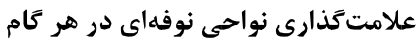

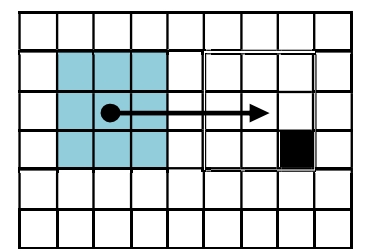

الف: علامت كذارى بيكسل هاى نوفهاى

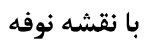

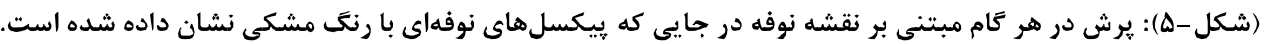

(Figure-5): The adaptive stride in CNN based on noise map. Noisy pixels are indicated by black color.

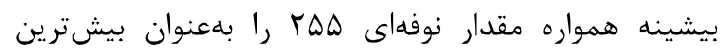

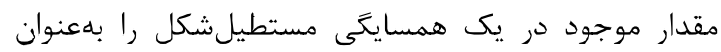

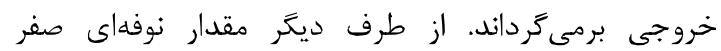

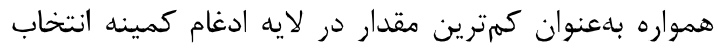

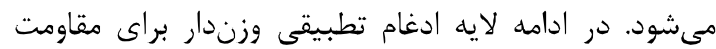
CNN برابر نوفه معرفى مىشود.

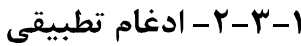

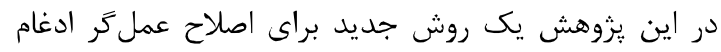

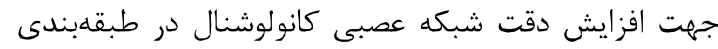

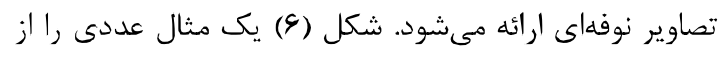

r-r- لايه ادغام تطبيقى وزندار در برابر نوفه

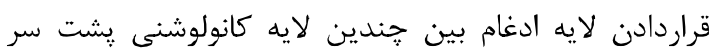

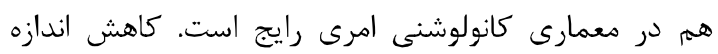

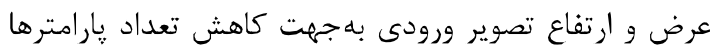

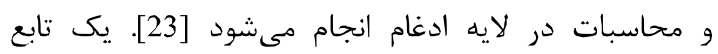
ادغامكننده، خروجى شبكه در يك مكان خاص را با با خلاصئ

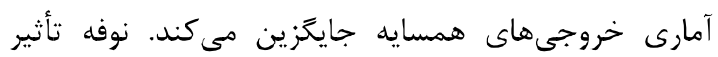

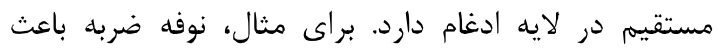

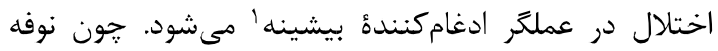

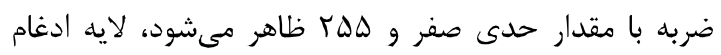

${ }^{1}$ Max Pooling 
نوفهاى بارها استفاده كرد. در شكل (A) روش يِيشنهادى در

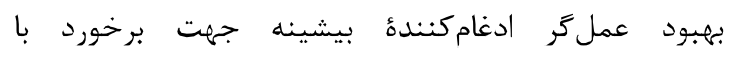

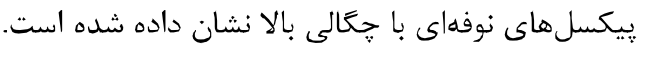

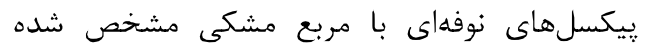

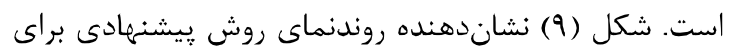
بهبود عمل ₹ر ادغامكننده بيشينه جهت حذف نوفه است. در اين روندنما يك سويج تعبيه شده است كه انتخاب روش رون

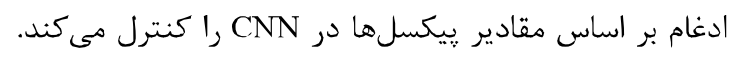

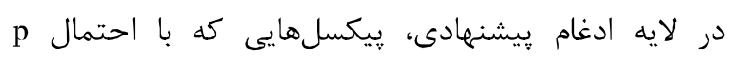
بهوسيلة نوفه تخريبشده باشند، با همان احتمال در عمليات

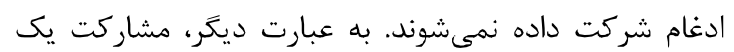

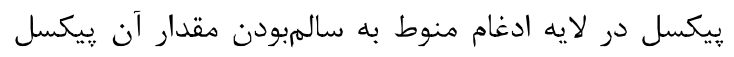
است. آر يك يِيكسل بهاحتمال نوفهاى است، در لايه ادغام

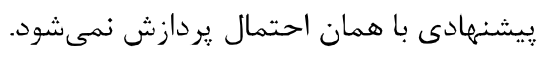

عمل كر ادغام بيشينه نشان مىدهد. براى حذف نوفه در لايه

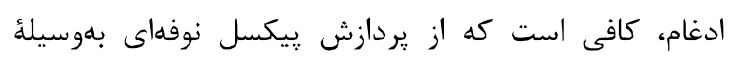

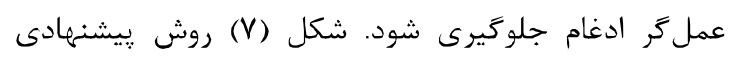

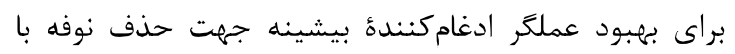

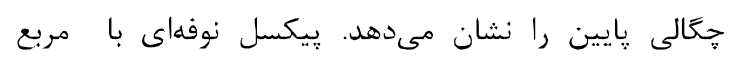
مشكى مشخص شده است. همانطور كه در تصوير مشاهده

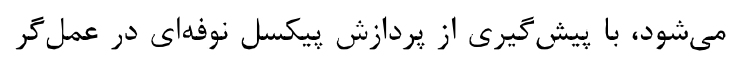

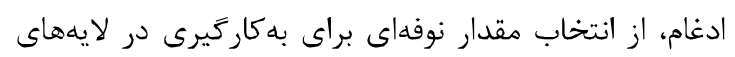

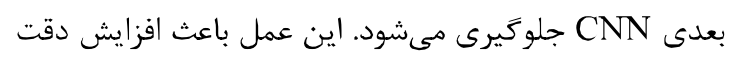
طبقهبندى مىشود.

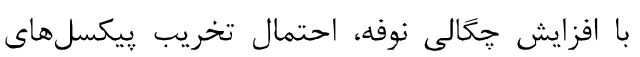
كل ينجره در تصوير وجود دارد. با توجه به اينكه عمل ادغام קندينبار در لايههاى شبكه عصبى كانولوشنال تكرار

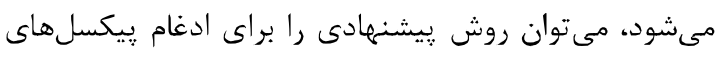

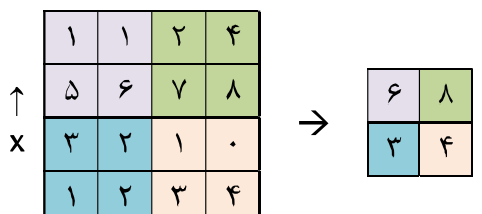

$y \rightarrow$

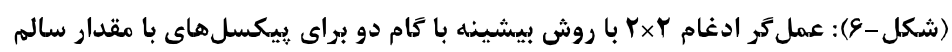

(Figure-6): $2 \times 2$ max pooling operator with a stride of 2 pixels for uncorrupted pixels

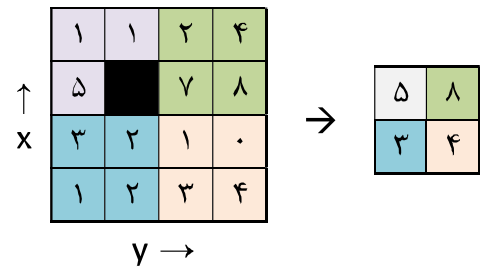

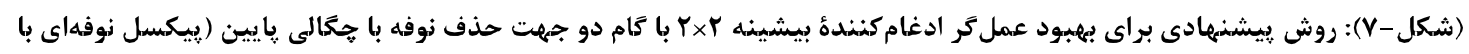

$$
\text { مربع مشكى مشخص شده است }
$$

(Figure-7): The proposed method for improving $2 \times 2$ max pooling operator with a stride of 2 pixels to remove low density noise (Black squares indicate the noisy pixels)

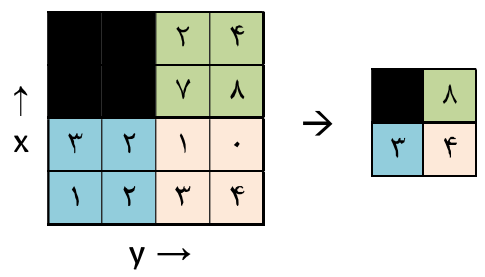

(شكل-1): رهيافت معرفى شده براى بهبود عمل كر ادغام كنندة بيشينه جهت حذف نوفه با جمكالى بالا

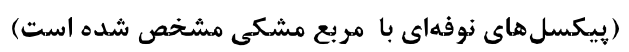

(Figure-8): The proposed method for improving the max pooling operator to remove high density noise (Black squares indicate the noisy pixels) 


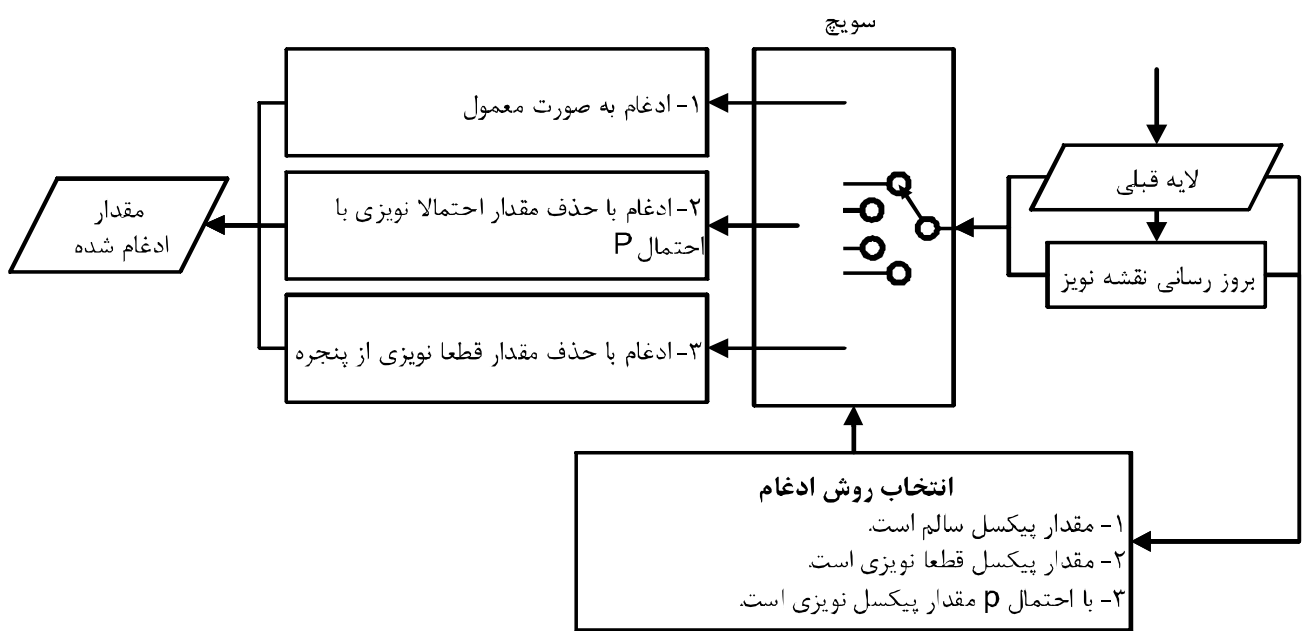

(شكل - (9): روندنماى روش بيشنهيهادى براى بيهبود عمل كر ادغام كنندة بيشيذه جهت حذف نوفه

(Figure-9): The workflow of the proposed method for improving the max-pooling operator to remove noise

بيشترى در برابر نوفه خواهد داشت. وزن صفر براى مقادير

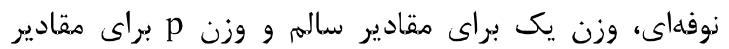

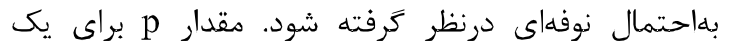

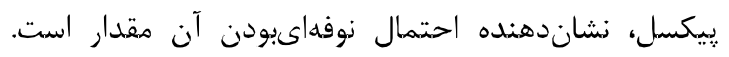

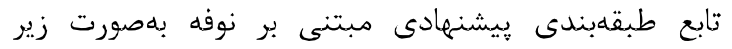
تعريف مىشود: $f(x)=w^{\prime} X(\alpha u+\beta q)+b, \alpha \in \mathbb{R}, \beta \in \mathbb{R}$

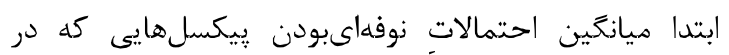

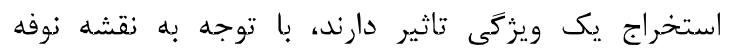

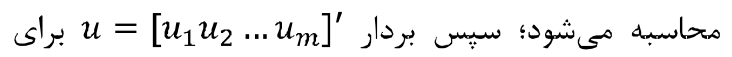

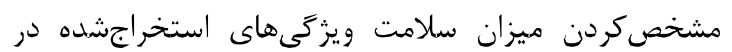

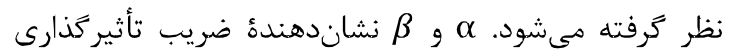
نوفه بر تابع طبقهبندى است.

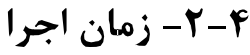

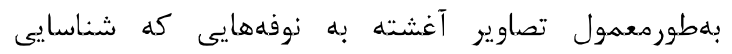
بيكسل نوفهاى ممكن باشد، مانند نوفه ضربه، بيكسله إنهاى

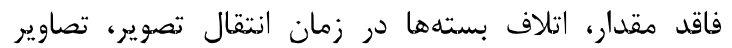
تخريبشده با دادهاى يرت، تصاوير دست كارىشده، در دو ناف

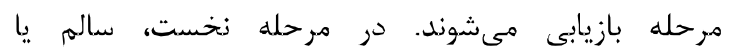
نوفهائودن هقدار بيكسل شناسايى داده مىشود. اصلاح

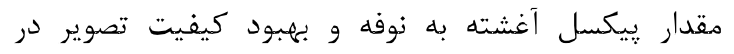
مرحله دوم انجام مىشود [8]-[10], [20] در اين مقاله، مرحله نخست يا شناسايى نوفه با با تهيه

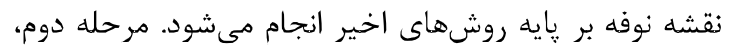

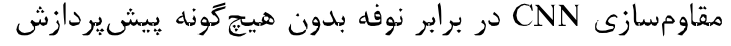

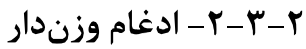

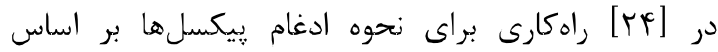

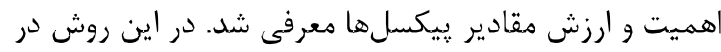

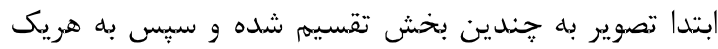
از مقاديرى كه قرار است، ادغام شوند، يكى وزن اختصاص إد إنداص داده مىشود. عمليات ادغام بر اساس وزن هريك از مقادير الداري

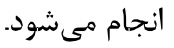

با توجه به تصوير Iا، ابتدا ويزّكىهاى محلى بال fوشهايى هانند SIFT استخراج و بلصورت بيان مىشود كه m نشاندهنده تعداد ناحيههاى محلى در

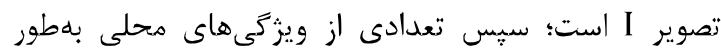
تصادفى بهوسيله خوشهبندى k-means انتخاب مىشوند؛ بنابراين هر تصوير را مىتوان با يكى ماتريس

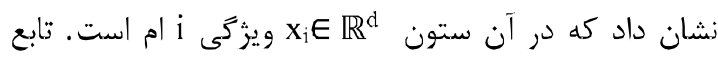
طبقهبندى در [24] بهصورت زير تعريف شده است: $f(x)=w^{\prime} X q+b$

كه ' بردارى است كه وزن ويزگیىهاى هحلى را معين هى كند و طبقهبند هستند.

براى بهبود روش [24] براى هقاومسازى CNN در هر

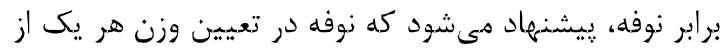
مقادير يِيكسلها در ادغام تطبيقى مشاركت داشته باشد؛

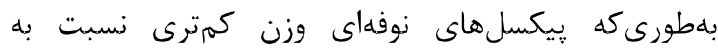
يِكسلهاى سالم براى انجام عمليات ادغام داشته باشند. با نيا

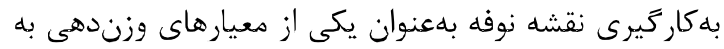

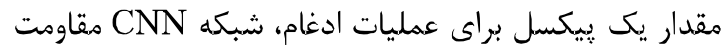


براى يادگيرى باناظر در مدل بيشنهادى بهكار زرفته شده است.

براى شبيهسازى انواع نوفهها از توزيع يكنواخت

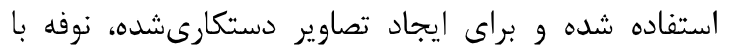

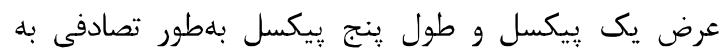
تصوير اضافه شده است. همجنين براى ساخت تصاوير تخريبشده با دادهاى زيرت، دايرههاى توير با شعاع دو

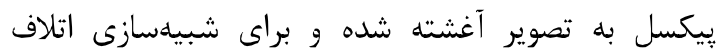

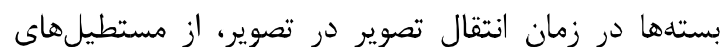

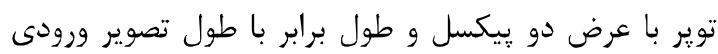

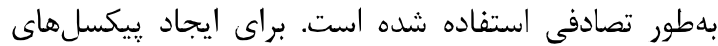

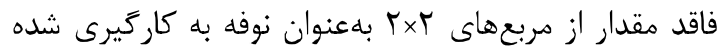

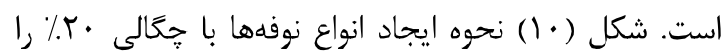
نشان مي دهد.

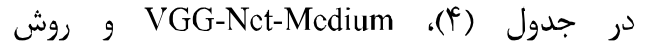
بيشنهادى براى طبقهبندى تصاوير نوفهاى مقايسه شدهاند.

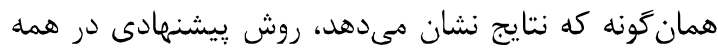

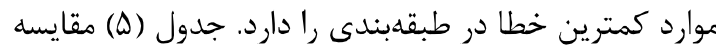
طبقدبندى روش بيشنهادى و VGG-Net-Slow براى تصاوير

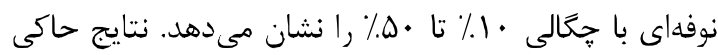
از برترى روش بيشنهادى در طبقهبندى تصاوير نوفهاى

براى مقايسه مدل ييشنهادى از يايخاهداده MNIST

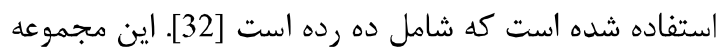
داده از هفتادهزار تصوير تشكيل شده كه شصتهزار نمونه سالم براى آموزش و دهززار نمونه نوفهاى براى آزمون

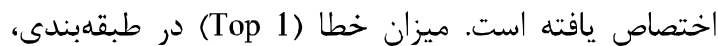

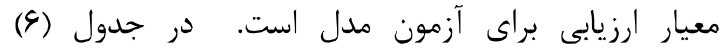

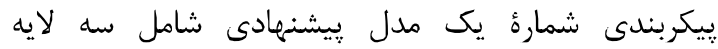

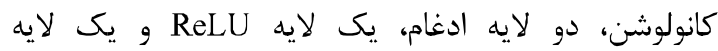
بهطور كامل متصل نشان داده شده است. ييكربندى شماره

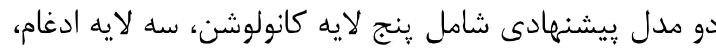

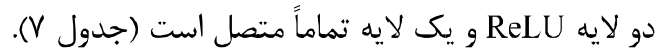

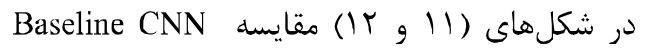

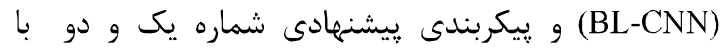
يارامترهاى n=5 و n=7 براى طبقلهبندى تصاوير نوفهاى

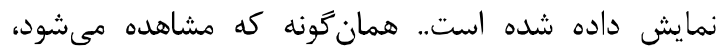
كمترين خطا در شكل (11) با پييكربندى شماره دو با ابعاد

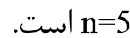

است. با توجه به جدول (1) روش ييشنههادى در تمام مراحل

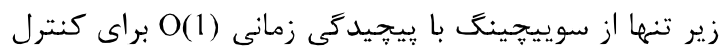
ييكسل هاى نوفهاى استفاده مي كند.

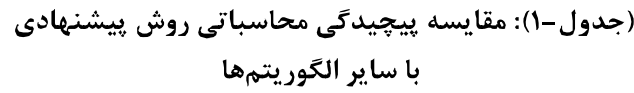

(Table-1): Comparison of computational complexity for the proposed method with other algorithms

\begin{tabular}{|c|c|}
\hline يِيجيدگى زمانى & الكوريته - الم \\
\hline$O(1)$ & روش بيشنهادى (يرش تطبيقى) \\
\hline $\mathrm{O}(1)$ & روش ييشنهادى (لايه ادغام تطبيقى) \\
\hline $\mathrm{O}(1)$ & روش بِيشنهادى (لايه ادغام وزندار) \\
\hline $\mathrm{O}(\mathrm{n})$ & فيلتر ميانه \\
\hline $\mathrm{O}(\mathrm{n})$ & الكوريتم MDBUTMF] \\
\hline$O(n)$ & روش زنخ و همكاران \\
\hline $\mathrm{O}(\mathrm{n})$ & روش جفر و همكاران \\
\hline
\end{tabular}

\section{" ش- شبيهسازى}

VGG-Net براى مقايسه روش ريشنهلادى از مدل آموزشديده [25] با معمارى medium و slow استفاده شده ميد

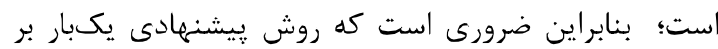
يايه معمارى medium و يكبار بر يايه معمارى slow

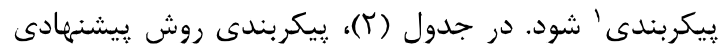
بر קِيه مدل VGG-Net-Medium قابل مشاهده است. جدول

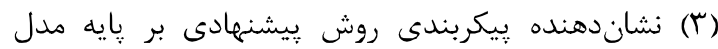
VGG-Net-Slow ينج لايه كانولوشن و سه لايه تماماً متصل، اجزاى تشكيلدهنده مدل زيشنهادى است. تصاوير ورودى بهاندازة

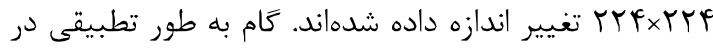
برابر نوفه است. جهت نرمالسازى از LRN² استفاده شده است [25]. از ادغام تطبيقى وزندار در لايه ادغام بهكارگيرى اسل

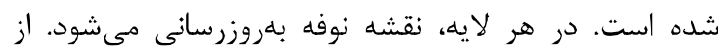
تابع فعالساز RELU در همه لايهها به غير از آخرين لايه لايه

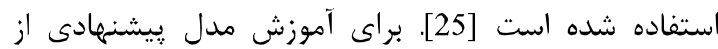
يايخاهداده ILSVRC-2012 استفاده شده [26] كه شامل

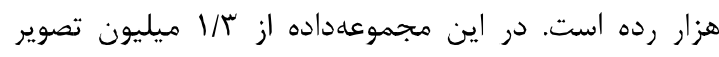
براى آموزش و ينجاه هزار تصوير براى اعتبارسنجى استفاده

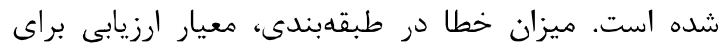

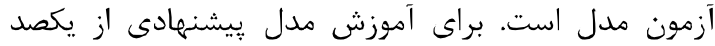

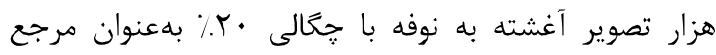
الكوى نوفه با توزيع يكنواخت استفاده شده است. نقشه نوفه به بـه

${ }^{1}$ Configuration

${ }^{2}$ Local Response Normalization (LRN)

${ }^{3}$ REctification Linear Unit 

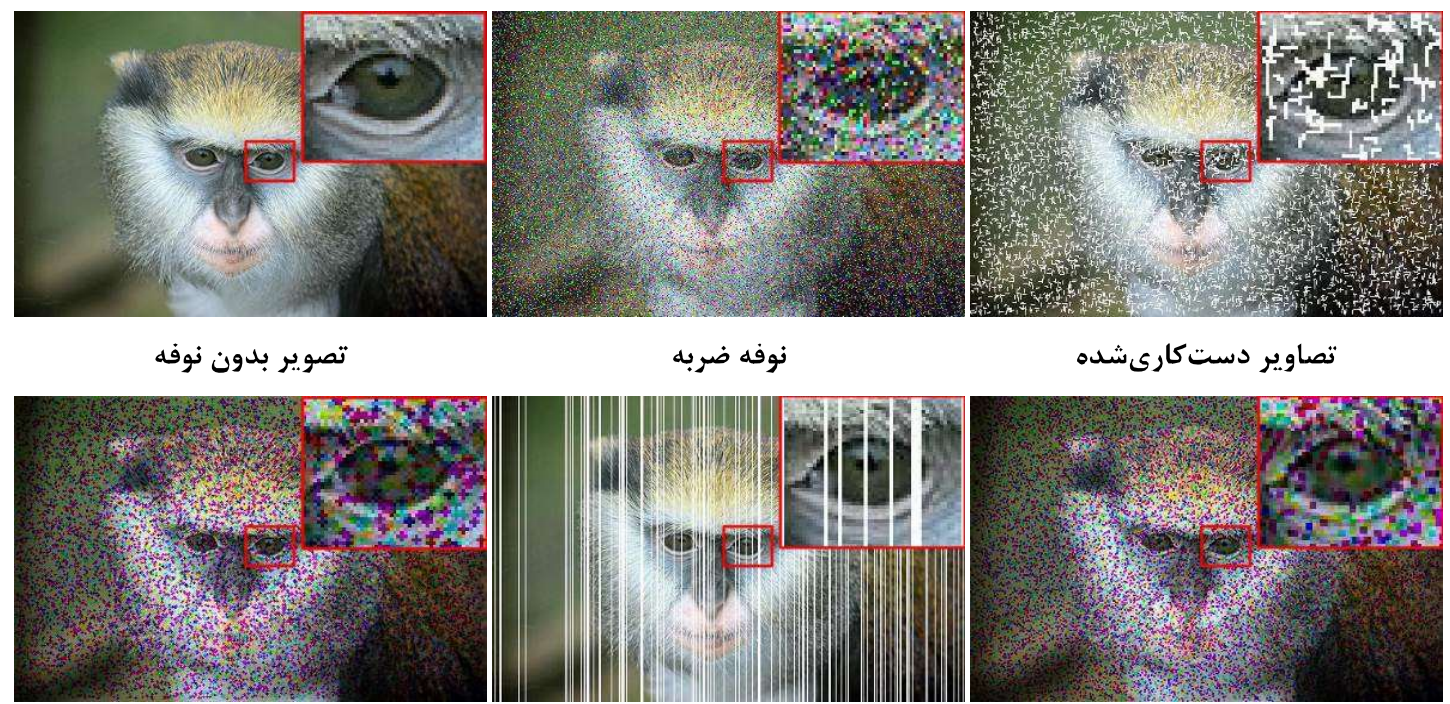

تصاوير دستكارىشده

تصاوير تخريب شده با دادههاى يرت

اتلاف بستهها در زمان انتقال

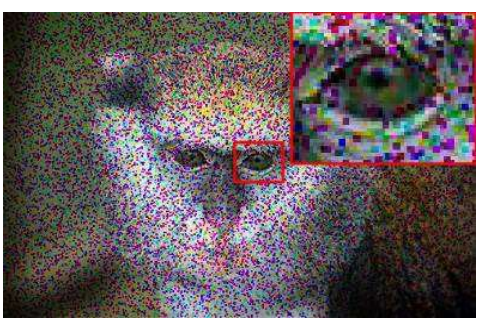

(شكل -•()): نحوه شبيهسازى انواع نوفهها. تصاوير بزرتىنمايى شدهاند.

(Figure-10): The creation of a variety of noises with $20 \%$ density. Images are magnified.

(جدول-Y): ييكربندى روش پيشنهادى بر يايه VGG-Net-Medium

(Table-2): Configuration of the proposed NR-CNN based on the VGG-Net-Medium model

\begin{tabular}{|c|c|c|c|c|c|c|}
\hline Layer & VGG-Net-Medium & Proposed & Size & Number & Stride & Pading \\
\hline 1 & - & Noise detection & - & - & - & - \\
\hline$r$ & Input & Input & - & - & - & - \\
\hline r & Convolution & convolution & $V \times V$ & 99 & r & . \\
\hline$f, \omega$ & ReLU, LRN & ReLU, LRN & - & - & - & - \\
\hline 4 & Max pooling & Adaptive max pooling & $r \times r$ & - & r & - \\
\hline$\checkmark$ & Convolution & Convolution & $\Delta \times \Delta$ & ras & r & 1 \\
\hline$\wedge, 9$ & ReLU, LRN & ReLU, LRN & - & - & - & - \\
\hline 1 . & Max pooling & Adaptive max pooling & $r \times r$ & - & r & - \\
\hline 11 & Convolution & Convolution & $r \times r$ & $\Delta I T$ & 1 & 1 \\
\hline ir & ReLU & ReLU & - & - & - & - \\
\hline Ir & Convolution & Convolution & $r \times r$ & $\Delta / T$ & 1 & 1 \\
\hline if & ReLU & ReLU & - & - & - & - \\
\hline 10 & Convolution & Convolution & $r \times r$ & DIT & 1 & 1 \\
\hline 19 & ReLU & ReLU & - & - & - & - \\
\hline iv & Max pooling & Adaptive max pooling & $r \times r$ & - & r & - \\
\hline 11 & Fully connected & Fully connected & - & $4 \cdot 99$ & 1 & - \\
\hline 19 & ReLU & ReLU & - & - & - & - \\
\hline$r \cdot$ & Fully connected & Fully connected & - & 4.99 & 1 & - \\
\hline M & ReLU & ReLU & - & - & - & - \\
\hline Tr & Fully connected & Fully connected & - & $1 \cdots$ & 1 & - \\
\hline rr & Loss function & loss function & - & - & - & - \\
\hline
\end{tabular}


(جدول - "r): بيكربندى روش بيشنهادى بر بايه VGG-Net-Slow

(Table-3): Configuration of the proposed NR-CNN based on the VGG-Net-Slow model

\begin{tabular}{|c|c|c|c|c|c|c|}
\hline Layer & VGG-Net-Slow & Proposed & Size & Number & Stride & Pading \\
\hline 1 & - & Noise detection & - & - & - & - \\
\hline$r$ & Input & Input & - & - & - & - \\
\hline r & Convolution & convolution & $V \times V$ & 99 & r & • \\
\hline$Y, \Delta$ & ReLU, LRN & ReLU, LRN & - & - & - & - \\
\hline$\varepsilon$ & Max pooling & Adaptive max pooling & $r \times r$ & - & r & - \\
\hline V & Convolution & Convolution & $\Delta \times \Delta$ & TDS & 1 & • \\
\hline$\wedge$ & ReLU & ReLU & - & - & - & - \\
\hline 9 & Max pooling & Adaptive max pooling & $r \times Y$ & - & r & $\cdot$ \\
\hline 1. & Convolution & Convolution & $r \times r$ & $\Delta I T$ & 1 & 1 \\
\hline 11 & ReLU & $\mathrm{ReLU}$ & - & - & - & - \\
\hline ir & Convolution & Convolution & $r \times r$ & $\Delta I T$ & 1 & 1 \\
\hline ir & ReLU & RcLU & - & - & - & - \\
\hline if & Convolution & Convolution & $r \times r$ & $\Delta I T$ & 1 & 1 \\
\hline 10 & ReLU & ReLU & - & - & - & - \\
\hline 18 & Max pooling & Adaptive max pooling & $r \times r$ & - & r & • \\
\hline iv & Fully connected & Fully connected & - & $4 \cdot 98$ & 1 & - \\
\hline 11 & ReLU & ReLU & - & - & - & - \\
\hline 19 & Fully connected & Fully connected & - & 4.94 & 1 & - \\
\hline$r \cdot$ & ReLU & ReLU & - & - & - & - \\
\hline rI & Fully connected & Fully connected & - & $1 \cdots$ & 1 & - \\
\hline rt & Loss function & loss function & - & - & 1 & • \\
\hline
\end{tabular}

(جدول-f) مقايسه روش وِيشنهادى و VGG-Net-Medium براى طبقهبندى تصاوير نوفهاى با معيار خطا (Top 5 (ج)

(Table-4): The classification performance (Top-5 error) of noisy images using VGG-Net-Medium and proposed NR-CNN

\begin{tabular}{|c|c|c|c|c|c|c|}
\hline \multicolumn{3}{|c|}{ روش ״يشنهادى } & \multicolumn{3}{|c|}{ VGG-Net-M } & نوع نوفه \\
\hline$\Delta \%$ & $1 \cdot \%$ & $r \cdot \%$ & $\Delta \%$ & $1 \cdot \%$ & $r \cdot \%$ & \\
\hline$\cdot / 14$ & $\cdot / 49$ & $\cdot / r V$ & $\cdot / r T$ & $\cdot / 4 \Delta$ & .199 & نوفه ضربه \\
\hline$\cdot / r \Delta$ & $\cdot / r \wedge$ & $\cdot / \mu F$ & $\cdot / \mu \cdot$ & $\cdot / r \wedge$ & $\cdot|\Delta|$ & تصاوير دستكارىشده \\
\hline$\cdot / K F$ & $\cdot / T V$ & . Tr & $\cdot / r \Lambda$ & $\cdot / 48$ & $\cdot 10$ & تصاوير تخريب شده با دادهاى يرت \\
\hline$\cdot / 48$ & $\cdot / \mu 1$ & $\cdot / 49$ & $\cdot / \pi \Delta$ & $\cdot / 4 V$ & $\cdot 190$ & اتلاف بستهها در زمان انتقال تصوير \\
\hline$\cdot / \pi$ & $\cdot / T V$ & $\cdot / \pi 1$ & $\cdot / 49$ & $\cdot / \mu r$ & $\cdot / 49$ & ييكسل هاى فاقد مقدار \\
\hline
\end{tabular}

(جدول -ه): مقايسه روش ييشنهادى و VGG-Net-Slow براى طبقهبندى تصاوير نوفهاى با معيار خطا (Top 5 (T)

(Table-5): The classification performance (Top-5 error) of noisy images using VGG-Net-Slow and proposed NR-CNN

\begin{tabular}{|c|c|c|c|c|c|c|}
\hline \multicolumn{3}{|c|}{ روش بيشنهادى } & \multicolumn{3}{|c|}{ VGG-Net-S } & نوع نوفه \\
\hline$\Delta \%$ & $1 \cdot \%$ & $r \cdot \%$ & $\Delta \%$ & $1 \cdot \%$ & $r \cdot \%$ & \\
\hline.$/ T \Delta$ & $\cdot / \mu \cdot$ & $\cdot / \mathrm{r} \Lambda$ & $\cdot / / 1$ & $\cdot / F V$ & $\cdot / V T$ & نوفه ضربه \\
\hline$\cdot / K F$ & $\cdot / r \Lambda$ & • &.$/ 49$ & $\cdot / \mu \wedge$ & $\cdot|\Delta|$ & تصاوير دستكارىشده \\
\hline$\cdot / T F$ & $\cdot / T V$ & $\cdot / T r$ & $\cdot / 4 \lambda$ & $\cdot / 48$ & $\cdot|\Delta|$ & تصاوير تخريب شده با دادهاى يرت \\
\hline$\cdot / 74$ & •/Mt & $\cdot / \mu \wedge$ & $\cdot / r V$ & $\cdot / 49$ & $\cdot 190$ & اتلاف بستهها در زمان انتقال تصوير \\
\hline.$/ T t$ & $\cdot / T V$ & $\cdot / r 1$ & $\cdot / 48$ & $\cdot / \mu F$ & $\cdot / 4 q$ & ييكسل هاى فاقد مقدار \\
\hline
\end{tabular}


(جدول - ()): ييكر بندى شماره يك شبكه بيشنهادى

(Table-6): Configuration of the proposed NR-C.NN \#1

\begin{tabular}{|c|c|c|c|c|c|c|c|}
\hline Layer & BL-CNN & NR-CNN & Size & Number & Stride & $\begin{array}{c}\text { Pading } \\
\mathbf{n}=\Delta\end{array}$ & $\begin{array}{c}\begin{array}{c}\text { Pading } \\
n=V\end{array}\end{array}$ \\
\hline . & - & Noise detection & - & - & - & - & - \\
\hline 1 & Input & Input & - & - & - & - & - \\
\hline r & Convolution & Convolution & $n * n$ & $r \cdot$ & 1 & - & 1 \\
\hline$r$ & Max pooling & Adaptive Max pooling & $r * r$ & - & $r$ & . & . \\
\hline r & Convolution & Convolution & $\Delta * \Delta$ & $\Delta$. & 1 & . & . \\
\hline$\Delta$ & Max pooling & Max pooling & $r * r$ & - & r & · & . \\
\hline$\varsigma$ & Convolution & Convolution & $f * r$ & $\Delta \cdot$ & 1 & - & . \\
\hline$\checkmark$ & RcLU & ReLU & - & - & 1 & . & . \\
\hline$\Lambda$ & Fully connected & Fully connected & $1 * 1$ & 1. & 1 & • & . \\
\hline 9 & Loss function & loss function & - & - & - & - & - \\
\hline
\end{tabular}

(جدول - (Y) بيكربندى شماره دو شبكه بيشنهادى

(Table-7): Configuration of the proposed NR-CNN \#2

\begin{tabular}{|c|c|c|c|c|c|c|c|}
\hline \\
\hline Layer & BL-CNN & NR-CNN & Size & Number & Stride & $\begin{array}{c}\text { Pading } \\
\mathbf{n}=\boldsymbol{\Delta}\end{array}$ & $\begin{array}{c}\text { Pading } \\
n=\vee\end{array}$ \\
\hline - & - & Noise detection & - & - & - & - & - \\
\hline 1 & Input & Input & - & - & - & - & - \\
\hline r & Convolution & Convolution & $n * n$ & $r$. & 1 & + & 1 \\
\hline$r$ & Max pooling & Adaptive Max pooling & r*t & - & r & 1 & 1 \\
\hline r & Convolution & Convolution & $\Delta * \Delta$ & $\Delta \cdot$ & 1 & • & . \\
\hline$\Delta$ & Max pooling & Max pooling & $r * r$ & - & 1 & . & . \\
\hline 4 & RcLU & ReLU & - & - & 1 & . & · \\
\hline $\mathrm{V}$ & Convolution & Convolution & $f * f$ & s. & 1 & . & . \\
\hline$\wedge$ & Max pooling & Max pooling & t*t & - & 1 & • & . \\
\hline 9 & Convolution & Convolution & $r * r$ & $\Delta \cdots$ & 1 & . & . \\
\hline 1. & Max pooling & Max pooling & $r * r$ & - & 1 & . & . \\
\hline 11 & RcLU & $\mathrm{ReLU}$ & - & - & 1 & - & $\cdot$ \\
\hline ir & Fully connected & Fully connected & $1 * 1$ & 1. & 1 & . & - \\
\hline ir & Loss function & Adaptive loss function & - & - & - & - & - \\
\hline
\end{tabular}

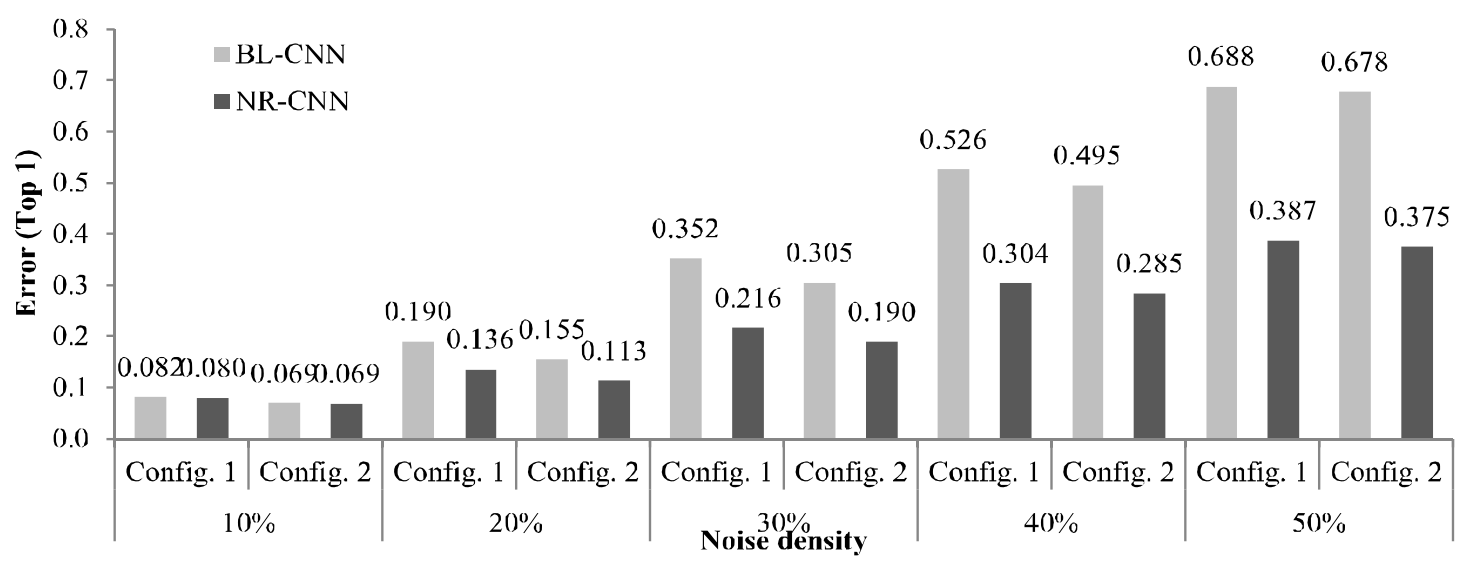

(شكل -1): مقايسه Baseline CNN با بيكربندى شماره يكى و دو روش ييشنهادى (n=5) و براى طبقهبندى تصاوير آغشته به نوفه ضربه (Figure-11): The classification performance of impulsive noisy images using configuration \#1 (n=5) 


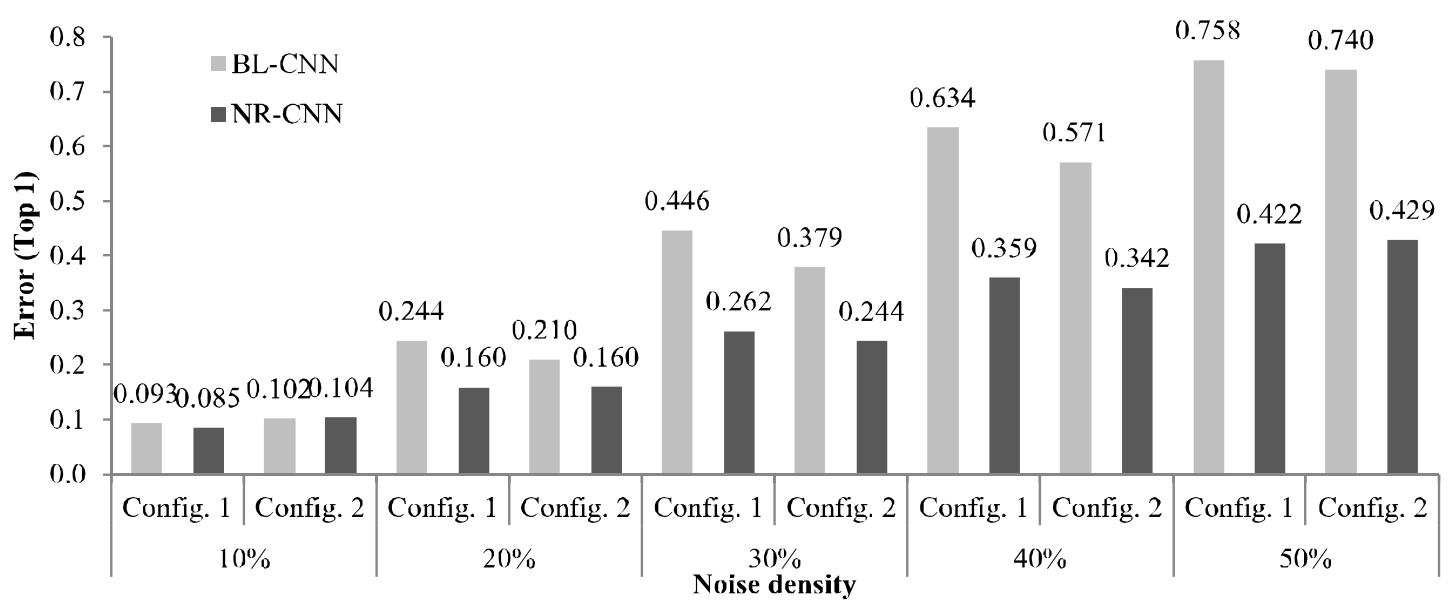

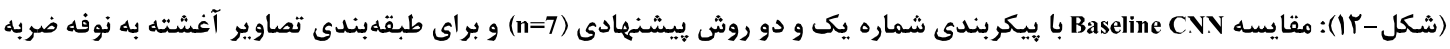

(Figure-12): The classification performance of impulsive noisy images using configuration \#2 ( $\mathrm{n}=7$ )

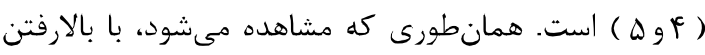

جكالى نوفه ضربه، دقت حذف نوفه كاهش مى

$P S N R=10 \log _{10}\left(\frac{255^{2}}{M S E}\right)$

$M S E=\frac{\sum_{i=1}^{N} \sum_{j=1}^{M}\left((Y 1(i, j)-Y 2(i, j))^{2}\right.}{M \times N}$

كه (i,j) بيكسل تصوير اصلى و Y1(i,j) بيكسل تصوير

اصلاح شده است. مختصات تصوير با i, مشخص ميىشود. M,N ו-r- مدت زمان به كارَّيرى روش هيشنهادى

زمان بازيابى تصوير آغشته به نوفه ضربه توسط الكوريتمهاى Wavelet ،27] k-ALS،[21] MDBUTMF،[21] MF ALOHA g [29] NLSPCA ،28] Blind،[16] TVL1،[16]

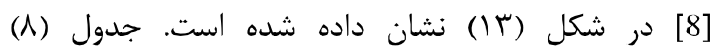

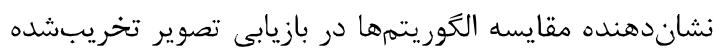

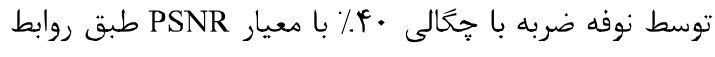

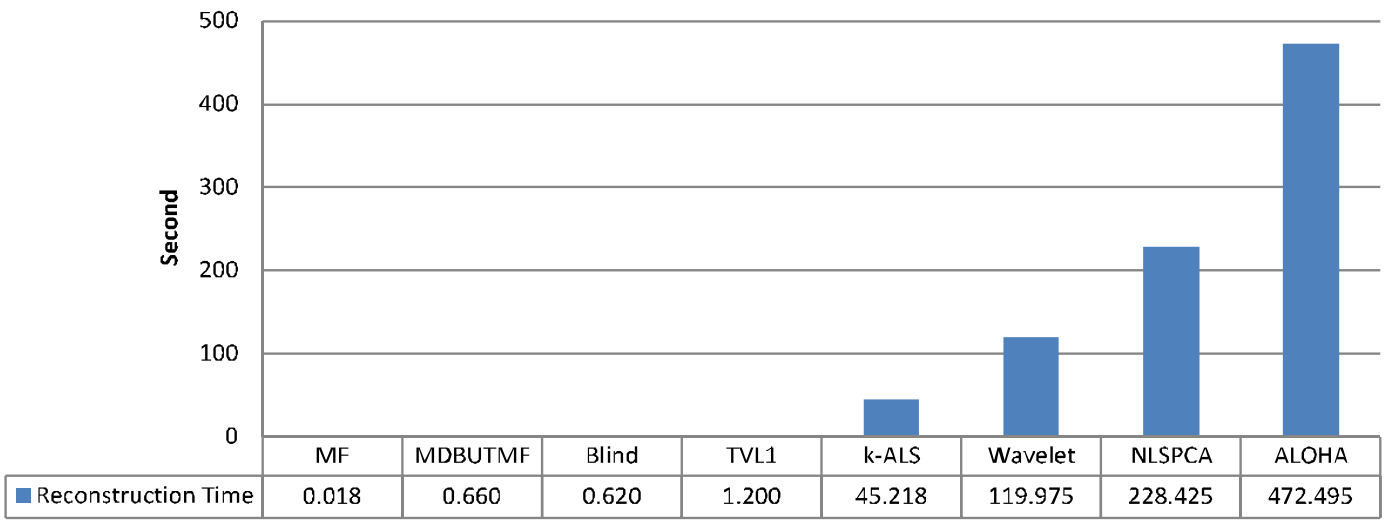

(شكل -rr): زمان بازيابى و بهبود تصاوير آغشته به نوفه ضربه توسط الكوريتمهاى مختلف [8

(Figure-13): Reconstruction time of the images corrupted by $40 \%$ impulse noise using different algorithms

مى دهد. موارد زير، روشهاى اخير براى رفع انواع نوفهها از تصاوير هستند: • تصاوير با بيكسلهاى فاقد مقدار: بازيابى نمونههاى

گممشده تصوير با استفاده از شاخص تشابه محدب [12] • تلفات ييكسلهاى تصاوير در ارسال و انتقال بستهها: يك

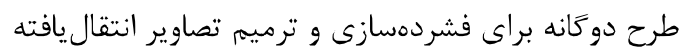

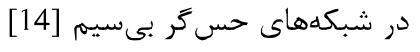

سريعترين روش اصلاح مقدار ييكسل آغشته به نوفه

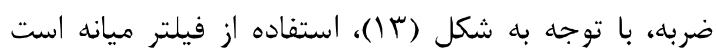
[8]. كمترين يِيجيدگى زمانى براى محاسبه مقدار ميانه با مرتبسازى سريع'، (O(n log(n) است. مقايسه بِيجيدگى زمانى روش يِشنهادى و سريعترين روش حذف نوفه ضربه مبتنى بر فيلتر ميانه، برترى روش پيشنهانى روس را نشان

${ }^{1}$ Quick Sort 


\section{5- References}

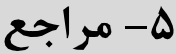

[1] Y. Hou, Z. Li, P. Wang, and W. Li, "Skeleton Optical Spectra-Based Action Recognition Using Convolutional Neural Networks," IEEE Trans. Circuits Syst. Video Technol., vol. 28, no. 3, pp. 807-811, 2018

[2] C. Ding and D. Tao, "Trunk-Branch Ensemble Convolutional Neural Networks for Video-Based Face Recognition," IEEE Trans. Pattern Anal. Mach. Intell., vol. 40, no. 4, pp. 1002-1014, 2018.

[3] L. C. Chen, G. Papandreou, I. Kokkinos, K. Murphy, and A. L. Yuille, "DeepLab: Semantic Image Segmentation with Deep Convolutional Nets, Atrous Convolution, and Fully Connected CRFs," IEEE Trans. Pattern Anal. Mach. Intell., vol. 40, no. 4, pp. 834-848, 2018.

[4] G. Lin, Q. Wu, L. Qiu, and X. Huang, "Image super-resolution using a dilated convolutional neural network," Neurocomputing, vol. 275, pp. 1219-1230, 2018.

[5] S. Yu, S. Jia, C. X.- Neurocomputing, and undefined 2017, "Convolutional neural networks for hyperspectral image classification," Elsevier, vol. 219, pp. 88-98, 2016.

[6] J. Yim and K. A. Sohn, "Enhancing the Performance of Convolutional Neural Networks on Quality Degraded Datasets," DICTA 2017 2017 Int. Conf. Digit. Image Comput. Tech. Appl., vol. 2017-Decem, pp. 1-8, 2017.

[7] Z. Zhang, D. Han, J. Dezert, and Y. Yang, "A new adaptive switching median filter for impulse noise reduction with pre-detection based on evidential reasoning," Signal Processing, vol. 147, pp. 173 189, 2018.

[8] K. H. Jin and J. C. Ye, "Sparse and low-rank decomposition of a hankel structured matrix for impulse noise removal," IEEE Trans. Image Process., vol. 27, no. 3, pp. 1448-1461, 2018.

[9] I. Turkmen, "The ANN based detector to remove random-valued impulse noise in images," J. Vis. Commun. Image Represent., vol. 34, pp. 28-36, 2016.

[10] S. Liang, S. Lu, J. Chang, and C. C. T. Lin, "A Novel Two-Stage Impulse Noise Removal Technique Based on Neural Networks and Fuzzy Decision," Ieeexplore.Ieee.Org, vol. 16, no. 4, pp. 863-873, 2008.

[11] V. K. Alilou and F. Yaghmaee, "Non-texture image inpainting using histogram of oriented gradients," J. Vis. Commun. Image Represent., vol. 48, pp. 43-53, 2017.

[12] A. Javaheri, H. Zayyani, and F. Marvasti, "Sparse recovery of missing image samples using a convex similarity index," Signal Processing, vol. 152, pp. 90-103, 2018.

$$
\begin{aligned}
& \text { • تصاوير تخريبشده با دادههاى يرت: يك رويكرد آمارى به } \\
& \text { مشكل بازگرداندن تصاوير آسيبديده [15] }
\end{aligned}
$$

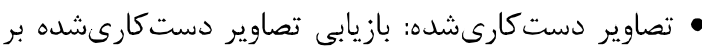

$$
\begin{aligned}
& \text { [18] VQ اساس شاخص } \\
& \text { با مقايسه روش يِيشنهادى و ساير روشهاى رفع نوفه } \\
& \text { براى انواع نوفهها، مشاهده مىشود كه زمان اجراى روش } \\
& \text { ييشنهادى كمتر ساير الكوريتهمها است؛ زيرا روش ييشنهادى } \\
& \text { بدون نياز به بيشيردازش براى حذف نوفه با مرتبه زمانى }
\end{aligned}
$$

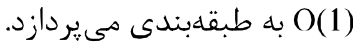

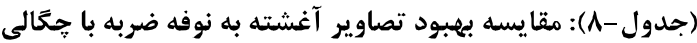

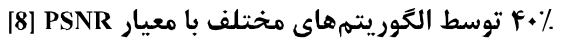

(Table-8): Reconstruction image PSNR using different algorithms for $20 \%$ and $40 \%$ impulse noise

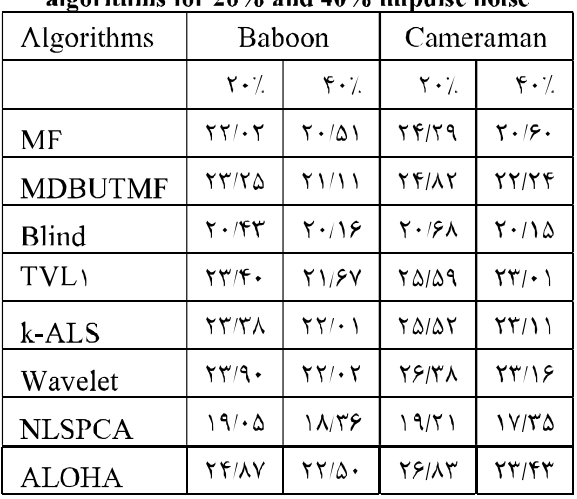

\section{p - نتيجه}

در اين مطالعه رويكردى جديد مبتنى بر ادغام تطبيقى يويا در برابر نوفه معرفى شد. اصلاح لايه ادغام براى مقاومسازى شبكه عصبى كانولوشنال در برابر انواع نوفهها، با وزندهى به مقادير קنجره مرجع، انجام شد. طبقلبندى تصاوير نوفهاى بدون نياز به ييشيردازش، مبتنى بر روش يِيشنهادى باعث تسريع فرايند طبقهبندى شده است. تصاوير آغشته به نوفه ضربه، تصاوير با پييكسلهاى فاقد مقدار، تلفات پيكسلهاى تصاوير در ارسال و انتقال بستهها، تصاوير تخريبشده با دادههاى يرت و تصاوير دستكارىشده مورد بررسى قرار

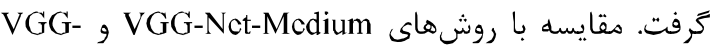
نشاندهنده نتايج بهتر روش پيشنهادى در Net-Slow طبقهبندى تصاوير نوفهاى است. نتايج شبيهسازى نشان مىدهد كه زمان اجراى روش بيشنهادى، بهجهت عدم نياز به يبشيردازش، كمتر از حالت با بيشيردازش براى حذف نوفه است. 
[26] O. Russakovsky, J. Deng, H. Su, J. Krause, S. Satheesh, S. Ma, Z. Huang, A. Karpathy, A. Khosla, M. Bernstein, A. C. Berg, and L. FeiFei, "ImageNet Large Scale Visual Recognition Challenge," Int. J. Comput. Vis., vol. 115, no. 3, pp. 211-252, 2015.

[27] Y. Wang, A. Szlam, and G. Lerman, "Robust Locally Linear Analysis with Applications to Image Denoising and Blind Inpainting," SIAM J. Imaging Sci., vol. 6, no. 1, pp. 526-562, 2013.

[28] M. Yan, "Restoration of Images Corrupted by Impulse Noise and Mixed Gaussian Impulse Noise using Blind Inpainting," SIAM J. Imaging Sci., vol. 6, no. 3, pp. 1227-1245, 2013.

[29] J. Salmon, Z. Harmany, C. A. Deledalle, and R. Willett, "Poisson noise reduction with non-local PCA," J. Math. Imaging Vis., vol. 48, no. 2, pp. 279-294, 2014
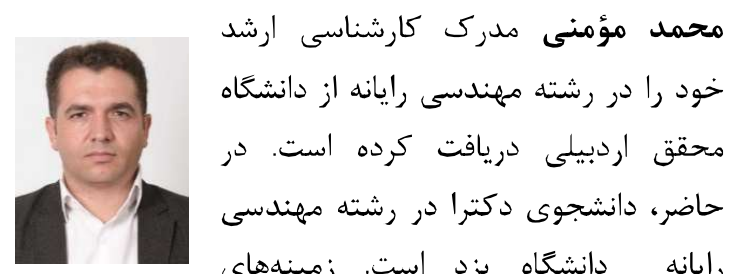
خود را در رشته مهيندسى رايانه از دانشعاه محقق اردبيلى دريافت كرده است. در حاضر، دانشجوى دكترا در رشته مهندسى

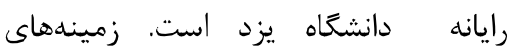
يخوهشى ايشان عبارتند از: يادخيرى عميق، بردازش تصوير و ياد كيرى ماشين. نشانى رايانامه ايشان عبارت است إن: mohamad.momeny@stu.yazd.ac.ir

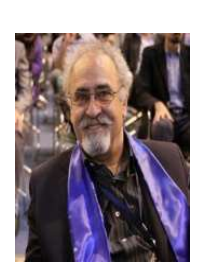

$$
\begin{aligned}
& \text { مهدى آقا صرام مدرك دكتراى خود را از } \\
& \text { دانشخاه ولز انگَلستان دريافت كرده است. } \\
& \text { در حال حاضر دانشيار زروه مهندسى رايانه } \\
& \text { دانشگاه يزد است. زمينههاى زئوهشى } \\
& \text { ايشان عبارتند از: يادكيرى هاشين، } \\
& \text { مهندسى نرمافزار، بيوانفورماتيك و داده كاوى. }
\end{aligned}
$$

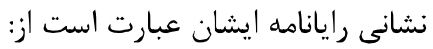

mehdi.sarram@yazd.ac.ir

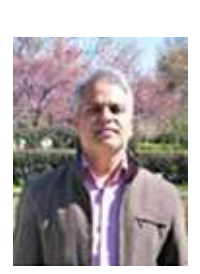

علىمحمد لطيف مدرى كارشناسى خود

را در رشته مهندسى برق از دانشعاه

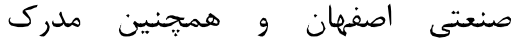

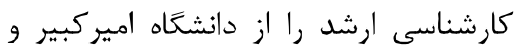

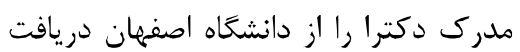

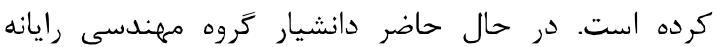

[13] D. Shabtay, N. Raviv, and Y. Moshe, "Video packet loss concealment detection based on image content," Eur. Signal Process. Conf., 2008.

[14] G. Nikolakopoulos, P. Stavrou, D. Tsitsipis, D. Kandris, A. Tzes, and T. Theocharis, "A dual scheme for compression and restoration of sequentially transmitted images over Wireless Sensor Networks," Ad Hoc Networks, vol. 11, no. 1, pp. 410-426, 2013.

[15] R. G. Everitt and R. H. Glendinning, "A statistical approach to the problem of restoring damaged and contaminated images," Pattern Recognit., vol. 42, no. 1, pp. 115-125, 2009.

[16] B. Dong, H. Ji, J. Li, Z. Shen, and Y. Xu, "Wavelet frame based blind image inpainting," Appl. Comput. Harmon. Anal., vol. 32, no. 2, pp. 268-279, 2012.

[17] H. Li, W. Luo, and J. Huang, "Localization of Diffusion-Based Inpainting in Digital Images," IEEE Trans. Inf. Forensics Secur, vol. 12, no. 12, pp. 3050-3064, 2017.

[18] C. Qin, C. C. Chang, and K. N. Chen, "Adaptive self-recovery for tampered images based on VQ indexing and inpainting," Signal Processing, vol. 93, no. 4, pp. 933-946, 2013.

[19] K. Audhkhasi, O. Osoba, and B. Kosko, "Noiseenhanced convolutional neural networks," Neural Networks, vol. 78, pp. 15-23, 2016.

[20] I. F. Jafar, R. A. Alna'Mneh, and K. A. Darabkh, "Efficient improvements on the BDND filtering algorithm for the removal of high-density impulse noise," IEEE Trans. Image Process., vol. 22, no. 3, pp. 1223-1232, 2013.

[21] S. Esakkirajan, T. Veerakumar, A. N. Subramanyam, and C. H. PremChand, "Removal of High Density Salt and Pepper Noise Through Modified Decision Based Unsymmetric Trimmed Median Filter," IEEE Signal Process. Lett., vol. 18, no. 5, pp. 287$290,2011$.

[22] C. Guillemot and O. Le Meur, "Image Inpainting," IEEE Signal Process. Mag., no. JANUARY, pp. 127-144, 2014.

[23] J. Gu, Z. Wang, J. Kuen, L. Ma, A. Shahroudy, B. Shuai, T. Liu, X. Wang, G. Wang, J. Cai, and T. Chen, "Recent advances in convolutional neural networks," Pattern Recognit., vol. 77, pp. 354-377, 2018.

[24] Y. Liu, Y. M. Zhang, X. Y. Zhang, and C. L. Liu, "Adaptive spatial pooling for image classification," Pattern Recognit., vol. 55, pp. 58-67, 2016.

[25] K. Simonyan and A. Zisserman, "Very Deep Convolutional Networks for Large-Scale Image Recognition," 2014. 


$$
\begin{aligned}
& \text { دانشعاه يزد است. زمينههاى يزوهشى ايشان عبارتند از: } \\
& \text { يردازش تصوير، يادكيرى عميق و يادَيرى ماشين. }
\end{aligned}
$$

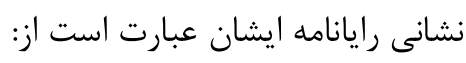

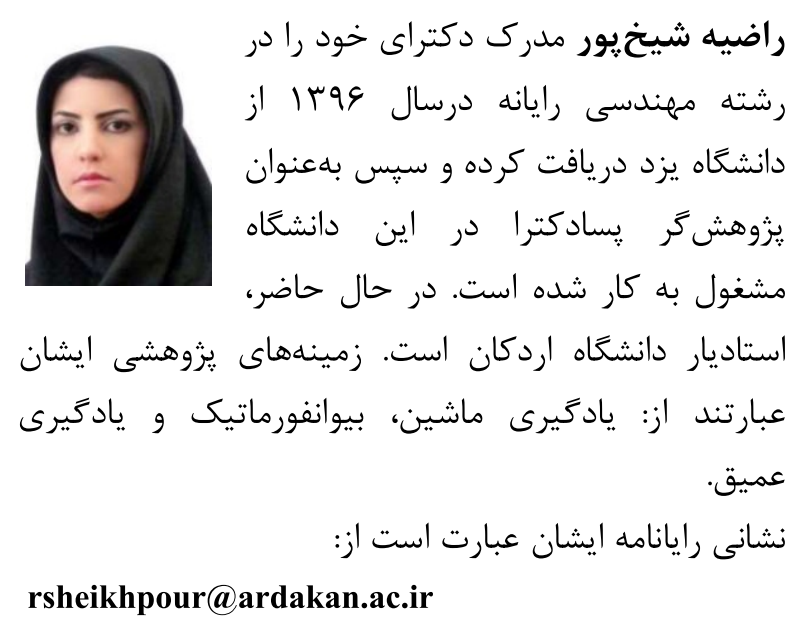


